\title{
Laser Illumination in Live Cell Microscopy: Scattering and Structured Illumination
}

\author{
Herbert Schneckenburger*, Verena Richter, Mathis Piper, and Michael Wagner \\ Aalen University, Institute of Applied Research, Beethovenstr. 1, 73430 Aalen, Germany \\ *e-mail: Herbert.schneckenburger@hs-aalen.de
}

\begin{abstract}
Two types of laser illumination in live cell microscopy with a focus on the sample or in the aperture plane of the microscope objective lens are distinguished. For the second case two examples are described, namely light scattering microscopy with angular resolution and Structured Illumination Microscopy (SIM) with two interfering laser beams. Appropriate applications include morphological studies of cells undergoing apoptosis and mitochondrial imaging with increased resolution. (C) 2017 Journal of Biomedical Photonics \& Engineering.
\end{abstract}

Keywords: Living cells; light scattering microscopy; super-resolution microscopy; structured illumination; SIM; apoptosis; mitochondrial imaging.

Paper \#3167 received 2 Mar 2017; revised manuscript received 4 Apr 2017; accepted for publication 7 Apr 2017; published online 12 Apr 2017. doi: 10.18287/JBPE17.03.010304. [Special Issue. Years in Biophotonics: 70th Anniversary of Prof. A.V. Priezzhev].

\section{References}

1. J. Pawley, Handbook of Biological Confocal Microscopy, Plenum Press, New York (1996).

2. R. H. Webb, “Confocal optical microscopy," Rep. Prog. Phys. 59, 427-471 (1996).

3. A. Ashkin, "Acceleration and Trapping of Particles by Radiation Pressure," Phys. Rev. Lett. 24(4), 156-159 (1970).

4. J. Huisken, J. Swoger, F. del Bene, J. Wittbrodt, and E. H. K. Stelzer, "Optical sectioning deep inside live embryos by SPIM," Science 305(5686), 1007-1009 (2004).

5. C. Dunsby, "Optically sectioned imaging by oblique plane microscopy", Opt. Express 16(25), 2306-2316 (2008).

6. H. Schneckenburger, "Total internal reflection fluorescence microscopy: technical innovations and novel applications," Curr. Opin. Biotechnol. 16(1), 13-18 (2005).

7. V. Richter, F. Voit, A. Kienle, and H. Schneckenburger, "Light scattering microscopy with angular resolution and its possible application to apoptosis," J Microsc. 257(1), 1-7 (2015).

8. B. Angres, H. Steuer, P. Weber. M. Wagner, and H. Schneckenburger, "A membrane-bound FRET-based caspase sensor for detection of apoptosis using fluorescence lifetime and total internal reflection microscopy," Cytometry 75A(5), 420-427 (2009).

9. M. G. L. Gustafsson, L. Shao, P. M. Carlton, C. J. R. Wang, I. N. Golubovskaya, W. Z. Cande, D. A. Agard, and J. W. Sedat, "Three-dimensional resolution doubling in wide-field fluorescence microscopy by structured illumination," Biophys. J. 94(12), 4957-4970 (2008).

10. R. Heintzmann, and C. Cremer, "Lateral modulated excitation microscopy: Improvement of resolution by using a diffraction grating," Proc. SPIE 3568, 185-196 (1999).

11. R. Förster, H.-W. Lu-Walther, A. Jost. M. Kielhorn, K. Wicker, and R. Heintzmann, "Simple structured illumination microscope setup with high acquisition speed by using a spatial light modulator," Opt. Express 22(17), 20663-20677 (2014).

12. M. Müller, V. Mönkemöller, S. Hennig, W. Hübner, and T. Huser, "Open-source image reconstruction of super-resolution structured illumination microscopy data in ImageJ," Nature Communications, 10980 (2016).

13. C. F. Bohren, and D. R. Huffman, Absorption and Scattering of Light by Small Particles, Wiley-Interscience Publications, New York (1998).

14. V. Richter, S. Bruns, T. Bruns, P. Weber, M. Wagner, C. Cremer, and H. Schneckenburger, "Axial tomography in live cell laser microscopy," J. Biomed. Opt. 22(9), 091505 (2017). 
15. S. W. Hell, and J. Wichmann, "Breaking the resolution limit by stimulated emission: stimulated-emissiondepletion fluorescence microscopy," Opt. Lett. 19(11), 1-3 (1994).

16. E. Betzig, G. H. Patterson, R. Sougrat, O. W. Lindwasser, S. Olenych, J. S. Bonifacino, M. W. Davidson, J. Lippincott-Schwartz, and H. F. Hess, "Imaging intracellular fluorescent proteins at nanometer resolution," Science 313(5793), 1642-1645 (2006).

17. M. J. Rust, M Bates, X. Zhuang, "Sub-diffraction-limit imaging by stochastic optical reconstruction microscopy (STORM)," Nat. Methods 3(10), 793-796 (2006).

18. C. Cremer, and B. R. Masters, "Resolution enhancement techniques in microscopy," Eur. Phys. J. H 38(3), 281-344 (2013).

19. H. Schneckenburger, P. Weber, M. Wagner, S. Schickinger, V. Richter, T. Bruns, W. S. L. Strauss, and R. Wittig, "Light exposure and cell viability in fluorescence microscopy," J. Microsc. 245(3), 311-318 (2012).

\section{Introduction}

In epi-illumination microscopy, e.g. fluorescence or scattering microscopy, a light source is most commonly focused to the plane of a sample. If a laser beam is used for Illumination, this creates a highly focused spot which may be used for laser scanning microscopy [1,2] or for laser micro-manipulation, e.g. in an optical tweezer system [3]. In some special illumination systems laser irradiation is separate from the microscope lens, i.e. it is perpendicular or occurs under a certain angle to the detection path. This kind of illumination has been realized in light sheet microscopy $[4,5]$ or in prism based total internal reflection fluorescence microscopy (TIRFM) [6]. Comparably little attention has been paid to epi-illumination in a conventional microscope, where the laser beam is focused to the aperture plane of the objective lens which is generally placed in its rear focal plane. Depending on the exact position of the focus this results in a parallel beam in the plane of the sample under a well-defined angle of illumination including super-critical angles in objective-type TIRFM [6]. The present paper describes two setups and applications of this illumination technique, namely light scattering microscopy with angular resolution and Structured Illumination Microscopy (SIM) with two interfering laser beams.

\section{Materials and Methods}

\subsection{Cells and samples}

For experiments of light scattering microscopy 3T3 murine fibroblasts were grown as monolayers in Dulbecco's modified Eagle medium supplemented with $10 \%$ fetal calf serum, pyruvate and antibiotics at $37^{\circ} \mathrm{C}$ and $5 \% \mathrm{CO}_{2}$. Apoptosis was induced by addition of $2 \mu \mathrm{M}$ staurosporine for 4 hours, as reported earlier [7]. The occurrence of apoptosis under these conditions was verified previously using a Förster Resonance Energy Transfer (FRET) based sensor for caspase-3 [8]. For experiments of Structured Illumination Microscopy (SIM) we used small clusters of human MCF-7 breast cancer cells cultivated in Dulbecco's MEM/Ham F-12 at $37^{\circ} \mathrm{C}$ and $5 \% \quad \mathrm{CO}_{2}$. Cells were incubated with the mitochondrial marker rhodamine $123(\mathrm{R} 123 ; 5 \mu \mathrm{M})$ for 30 minutes prior to rinsing with Earle's Balanced Salt
Solution and fluorescence microscopy using a Plan Neofluar 40×/1.30 oil immersion objective lens together with a cover slide. Microscope slides and standards were used for additional test experiments.

\subsection{Illumination}

Fig. 1 shows the difference between focused illumination of a sample, used e.g. in laser scanning microscopy or an optical tweezer system (Fig. 1a), and focused illumination of the aperture plane (Fig. 1b). In the first case (Fig. 1a) a lens $L_{2}$ focuses the light source to a field diaphragm FD at the entrance of the microscope which is in a conjugated plane of the sample $\mathrm{S}$. Then the tube lens $\mathrm{L}_{3}$ of the microscope creates a parallel beam which passes the aperture A of the microscope objective lens $\mathrm{L}_{4}$ and is finally focused on the sample $\mathrm{S}$. In the second case (Fig. 1b) a parallel beam passes the field diaphragm (FD) and is focused by the tube lens $\mathrm{L}_{3}$ to the aperture plane in the rear focus of the objective lens, thus generating a parallel beam in the plane of the sample. Assuming a beam diameter of about $15 \mathrm{~mm}$ in front of the tube lens L3 (with a typical focal length of $164 \mathrm{~mm}$ ), this results in a numerical aperture $\mathrm{A}_{\mathrm{N}}=0.045$ and finally in a spot diameter $\mathrm{d}=1.22 \lambda / \mathrm{A}_{\mathrm{N}} \approx 13 \mu \mathrm{m}$ in the aperture plane of the objective lens, thus permitting about 460 positions of the laser spot across a $6 \mathrm{~mm}$ aperture. Often telescopes consisting of the lenses $\mathrm{L}_{1}$ and $\mathrm{L}_{2}$ are used for illumination, but only in the second case the light beam is parallel at the entrance FD of the microscope, whereas in the first case a focus is created. A pinhole $\mathrm{P}$ within the telescope (i.e. between the lenses $\mathrm{L}_{1}$ and $\mathrm{L}_{2}$ ) may fulfill various purposes, e.g. filtering of laser modes or selection of well-defined diffraction orders from an optical grating.

Illumination with a focus in the aperture plane of the microscope objective lens according to Fig. 1b has been used for scattering experiments with angular resolution [7]. In this case the collimated (parallel) laser beam is deflected by a mirror under a variable angle prior to expansion by the telescope to a final diameter of about $15 \mathrm{~mm}$. In the present microscope (Axiovert 200M, Carl Zeiss Microsystems GmbH, Jena, Germany) the tube lens is replaced by an assembly of three lenses without any loss of aperture and resolution, so that an angular range of about $100^{\circ}$ can be covered by the microscope 
a

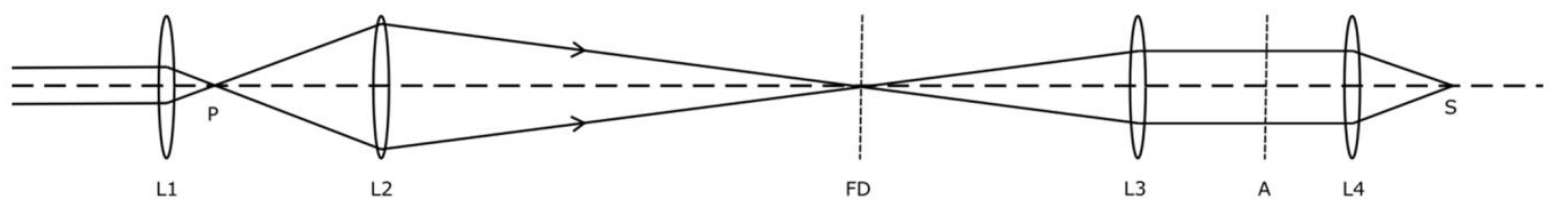

b

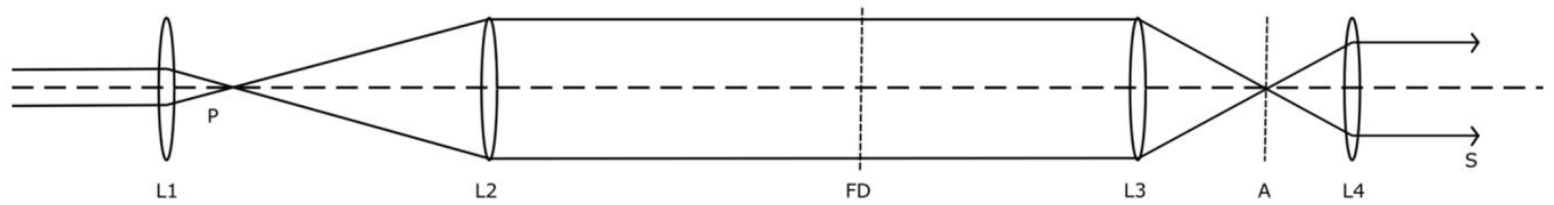

Fig. 1 Laser illumination in microscopy (a) focused on a sample $\mathrm{S}$ or (b) in the aperture plane A of the objective lens (lenses $\mathrm{L}_{1}, \mathrm{~L}_{2}, \mathrm{~L}_{3}$ and $\mathrm{L}_{4}$, field diaphragm FD, aperture $\mathrm{A}$ ).

with a resolution of $0.2-0.3^{\circ}$. Backscattered light can be collected by the same objective lens, collimated by a tube lens and focused by a so-called Bertrand lens to an exit pupil with a pinhole located in its centre, as depicted in Fig. 2. An angle of $180 \pm 1^{\circ}$ can thus be selected prior to light detection by a photomultiplier. Although backscattering can be measured in a large range of $130-230^{\circ}$ between the exciting and the scattered laser beam, an angular range of $190-220^{\circ}$ (or alternatively $140-170^{\circ}$ ) often revealed to be sufficient, so that specular reflection as well as large inclination angles (which might give some erroneous results) can be excluded.

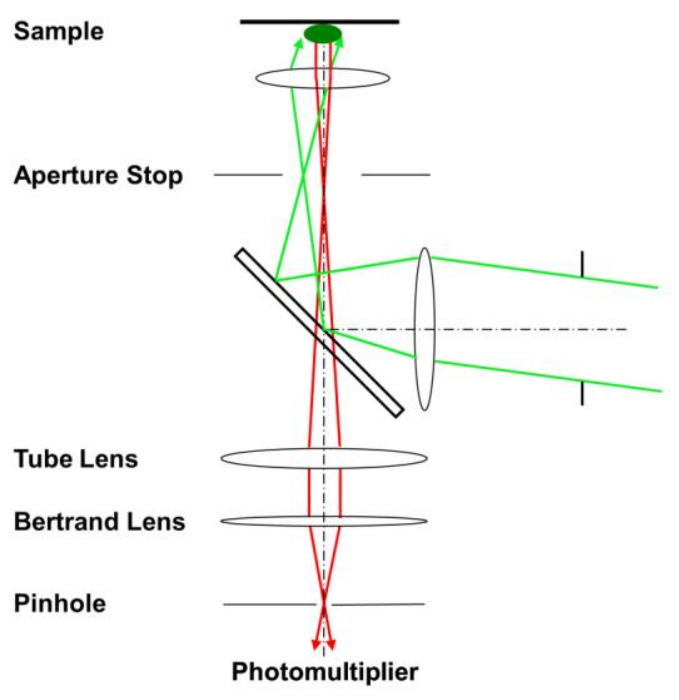

Fig. 2 Variable-angle light scattering microscopy. Entrance optics of the microscope may consist of a single tube lens (as indicated in the Fig.) or an assembly of several lenses (reproduced from [6] with modifications).

In Structured Illumination Microscopy (SIM) an interference pattern of at least two laser beams is created in the plane of the sample. In comparison with conventional fluorescence microscopy this gives higher spatial frequencies, broadens the optical transfer function (OTF) and increases resolution up to a factor 2 $[9,10]$. Two laser beams (e.g. the first diffraction orders of an optical grating or a spectral light modulator (SLM) upon blocking of $\mathrm{k}=0$ [11]) create an interference pattern at the entrance plane FD of the microscope. They are further focused at the edges of the microscope aperture, as depicted in Fig. 3a, to get optimum resolution. In the present case an angle $\alpha=1.02^{\circ}$ between the optical axis and each interfering laser beam was adjusted using a focal length $\mathrm{f}^{\prime}=150 \mathrm{~mm}$ for the lens $L_{2}$. This results in a grating constant of the interference pattern $d=\lambda / 2 \sin \alpha=13.71 \mu \mathrm{m}$ in the entrance plane FD and $343 \mathrm{~nm}$ in the plane of the sample at $40 \times$ diminution (Fig. 3 b) or $218 \mathrm{~nm}$ at $63 \times$ diminution by the objective lens $\mathrm{L}_{4}$ (in combination with the tube lens $\mathrm{L}_{3}$ ). From 3 rotation angles of the optical grating $\left(0^{\circ}, 60^{\circ}\right.$ and $\left.120^{\circ}\right)$ and 3 phases of the interference pattern in each case $(0,2 \pi / 3,4 \pi / 3)$ a resulting unstructured image with enhanced resolution (up to a factor 2 in comparison with conventional microscopy) can be calculated after Fourier transformation [12]. It should be mentioned that a disk of 6 pinholes can be placed in the common focus of the lenses $L_{1}$ and $L_{2}$ to select the $1^{\text {st }}$ diffraction orders of the optical grating or SLM, and that a $\lambda / 4$ plate together with a rotatable polarizer can be further introduced in the illumination rays to get interference in a welldefined plane of polarization.

\section{Applications}

An example for light scattering microscopy with angular resolution is given in Fig. 4 for the case of apoptosis of 3T3 murine fibroblasts. Light scattering is depicted from a small object field of 4-6 individual cells prior to (control) and subsequent to apoptosis (induced by addition of $2 \mu \mathrm{M}$ staurosporine to the culture medium for 4 hours). While the cells show pronounced changes in morphology after initiation of apoptosis (characterized by shrinking and almost 
a

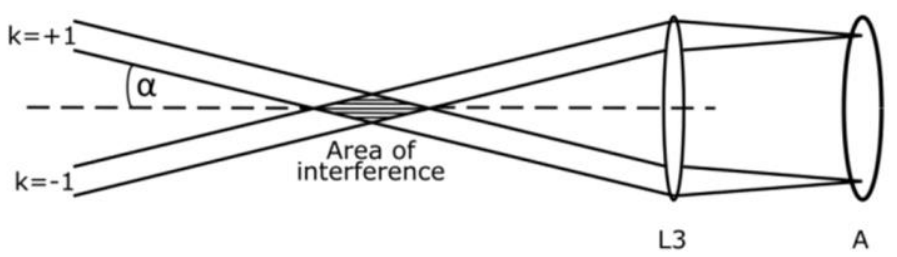

b

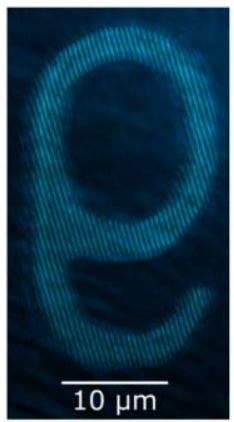

Fig. 3 (a) Interference of 2 laser beams used for Structured Illumination Microscopy (SIM) with laser foci in the aperture plane A; (b) Interference pattern on a microscope standard upon SIM by 2 interfering laser beams $\left(\mathrm{Ar}^{+}\right.$laser, $488 \mathrm{~nm}$ ) with a Plan Neofluar 40×/1.30 oil immersion objective lens using a modified Axiovert 200M microscope (Carl Zeiss).
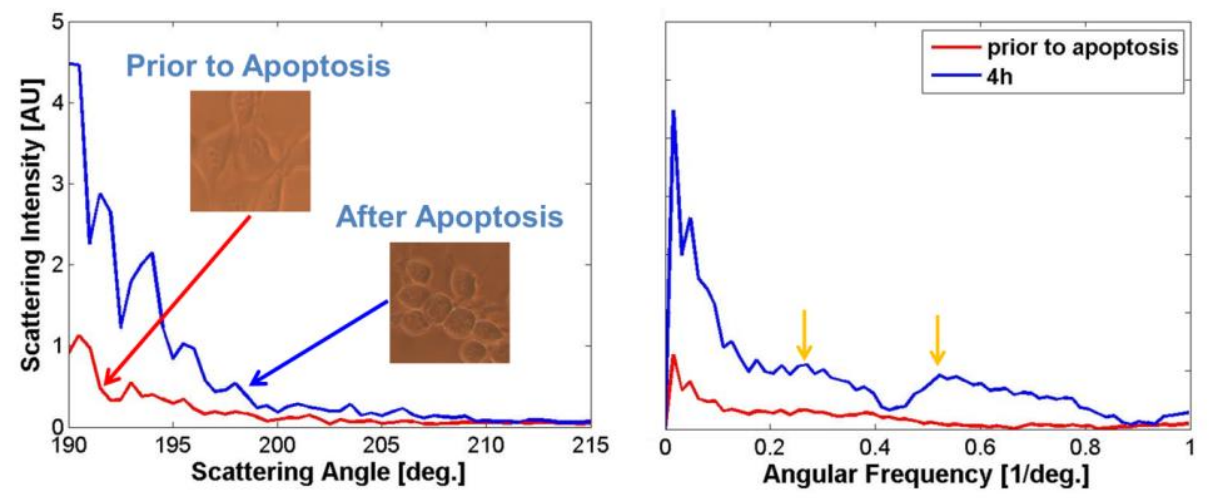

Fig. 4 Scattering intensity of 3T3 fibroblasts prior and subsequent to apoptosis (induced by $2 \mu \mathrm{M}$ staurosporine after 4h); angular resolution (left) and Fourier transform (right); scattering maxima are indicated by arrows.

a

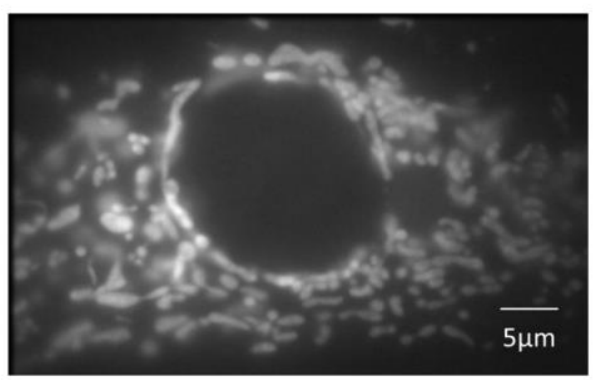

b

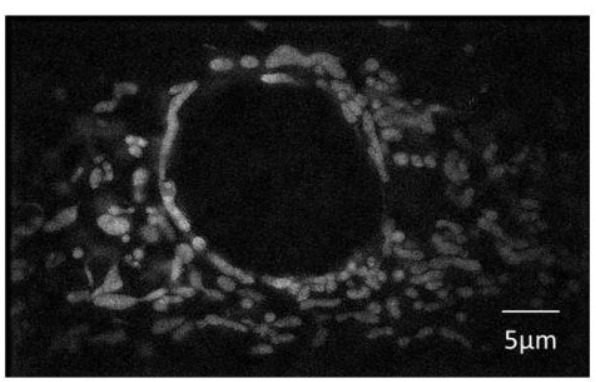

Fig. 5 MCF-7 breast cancer cells incubated with the mitochondrial marker R123 (5 $\mu$ M, 30 min.) excited by 2 interfering rays of an Ar+ laser (excitation wavelength: $\lambda_{\mathrm{ex}}=488 \mathrm{~nm}$; detection range: $\lambda_{\mathrm{D}} \geq 515 \mathrm{~nm}$; Plan Neofluar $40 \times / 1.30$ oil immersion microscope objective lens); (a) wide field image; (b) SIM image calculated from 9 structured images.

spherical shape, see insets of the Fig. 4a), the scattering intensity increases and exhibits pronounced oscillations, as described in the literature for Mie scattering [13]. After Fourier transformation maxima of the scattering function are resolved at angular frequencies close to 0 as well as around 0.3 and 0.5 oscillations per degree (Fig. 4b). A similar behaviour resulted from more than
15 other cell clusters (of 4 to 6 cells each) as well as a smaller number $(\leq 10)$ of multicellular spheroids [7]. In an attempt to describe this scattering behaviour, Mie scattering was simulated for a sphere of variable diameter and a refractive index $\mathrm{n}_{\mathrm{C}}=1.38$ (cell) surrounded by a medium of refractive index 1.33 , as well as for a sphere with a concentric inclusion of 
variable diameter and a refractive index $\mathrm{n}_{\mathrm{N}}=1.42$ (cell nucleus). Best results were obtained for a cell of $12.4 \mu \mathrm{m}$ diameter with a nucleus of $9.3 \mu \mathrm{m}$, which fairly corresponds to visual observation.

Fluorescence images of individual MCF-7 breast cancer cells incubated with the mitochondrial marker rhodamine 123 (R123; $5 \mu \mathrm{M}, 30 \mathrm{~min}$.) are depicted in Fig. 5. In both parts of this image a non-fluorescent nucleus is surrounded by mitochondria which appear as long-shaped fluorescent rods. While the wide-field image (Fig. 5a) shows some overlaying diffuse out-offocus fluorescence, the image calculated from 9 images with structured illumination (SIM; Fig. 5b) shows fluorescence only from the focal plane with improved resolution.

\section{Discussion}

Laser illumination with a focus in the rear focal plane of a microscope objective lens permits various applications with high angular resolution. In this paper we presented elastic light scattering as a method for detection of cell morphology, which may be suitable for studies of apoptosis as well as for measurement of infiltration of tumor cells into a host tissue [7]. In addition, angular resolution appears to be appropriate for objective-type Total Internal Reflection Fluorescence Microscopy (TIRFM) [6] or for dark field illumination in Raman microscopy.

Focusing of laser light in the rear focal plane of a microscope is also an appropriate method for interference microscopy, e.g. for creating an interference pattern in the plane of a sample which might be used for Structured Illumination Microscopy (SIM) with increased resolution. Lateral resolution cannot be determined quantitatively by the present experiments, but can be estimated from preliminary results of reference samples (fluorescent beads) and calculated from the sum of spatial frequencies $\mathrm{k}_{\mathrm{A}}=2 \mathrm{~A}_{\mathrm{N}} / \lambda$ according to the Abbe criterion (with the numerical aperture $A_{N}$ and the fluorescence wavelength $\lambda$ ) and $\mathrm{k}_{\mathrm{G}}=1 / \mathrm{d}$ of the optical grating (with the grating constant $d$ in the plane of the sample) [9]. In our case with $\mathrm{A}_{\mathrm{N}}=1.30, \lambda=520 \mathrm{~nm}$ and $\mathrm{d}=343 \mathrm{~nm}$ (with a $40 \times$ objective lens) we obtain $\mathrm{k}=\mathrm{k}_{\mathrm{A}}+\mathrm{k}_{\mathrm{G}}=0.0079 \mathrm{~nm}^{-1}$ corresponding to a resolution $1 / \mathrm{k}=126 \mathrm{~nm}$. A $63 \times$ objective lens would result in a grating constant $\mathrm{d}=218 \mathrm{~nm}$, a spatial frequency $\mathrm{k}=0.0096 \mathrm{~nm}^{-1}$ and a resolution of $104 \mathrm{~nm}$ which is by about a factor 2 better than the resolution resulting (only) from the Abbe criterion. Furthermore, in axial tomography [14] a sample can be rotated by variable angles, so that this very high resolution can be obtained in any direction of the sample. Presently a series of 9 structured images can be recorded with a light dose of about $10 \mathrm{~nJ} / \mu \mathrm{m}^{2}$ corresponding to the light dose after 10 seconds of solar exposure. This light dose is more than 1000 times lower than corresponding values obtained with other methods of super-resolution microscopy (e.g. STED [15], PALM [16], STORM [17] and related methods [18]) and allows living cells to be examined under non-phototoxic conditions [19] repeatedly over a longer time period or under variable angles of illumination.

\section{Acknowledgment}

The authors thank the Ministry of Science and Art Baden-Württemberg for funding the Project "MicroLife" and the Baden-Württemberg-Stiftung gGmbH for financing the project "Mistral". The authors also thank R. Heintzmann and R. Förster (IPHT Jena) as well as C. Cremer (IMB Mainz) for helpful discussions and C. Hintze for skillful technical assistance. 